\title{
Inadequate intake of nutrients essential for neurodevelopment in children with fetal alcohol spectrum disorders (FASD)
}

\author{
Anita J. Fuglestad, PhDa, Birgit A. Fink, BA ${ }^{\mathrm{a}}$, Judith K. Eckerle, MDa, Christopher J. Boys, \\ PhD $^{a}$, Heather L. Hoecker, BA ${ }^{b}$, Maria G. Kroupina, PhD ${ }^{a}$, Steven H. Zeisel, MD, PhD', \\ Michael K. Georgieff, MDa, and Jeffrey R. Wozniak, PhDa \\ aUniversity of Minnesota Twin Cities

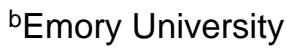 \\ cUniversity of North Carolina at Chapel Hill Nutrition Research Institute
}

\begin{abstract}
This study evaluated dietary intake in children with fetal alcohol spectrum disorders (FASD). Preclinical research suggests that nutrient supplementation may attenuate cognitive and behavioral deficits in FASD. Currently, the dietary adequacy of essential nutrients in children with FASD is unknown. Dietary data were collected as part of a randomized, doubleblind controlled trial of choline supplementation in FASD. Participants included 31 children with FASD, ages 2.5 - 4.9 years at enrollment. Dietary intake data was collected three times during the nine month study via interview-administered 24-hour recalls with the Automated Self-Administered 24-hour Recall. Dietary intake of macronutrients and 17 vitamins/minerals from food were averaged across three data collection points. Observed nutrient intakes were compared to national dietary intake data of children ages 2 - 5 years (What we Eat in America, NHANES 2007-2008) and to the Dietary Reference Intakes. Compared to the dietary intakes of children in the NHANES sample, children with FASD had lower intakes of saturated fat, vitamin D, and calcium. The majority ( $>50 \%)$ of children with FASD did not meet the Recommended Dietary Allowance (RDA) or Adequate Intake (AI) for fiber, n-3 fatty acids, vitamin D, vitamin E, vitamin K, choline, and calcium. This pattern of dietary intake in children with FASD suggests that there may be opportunities to benefit from nutritional intervention. Supplementation with several nutrients including choline, vitamin D, and $\mathrm{n}-3$ fatty acids, has been shown in animal models to attenuate the cognitive deficits of FASD. These results highlight the potential of nutritional clinical trials in FASD.
\end{abstract}

\section{Keywords}

Fetal alcohol (FAS, FASD); Nutrition; Dietary intake

(C) 2013 Elsevier Inc. All rights reserved

Address Correspondence: Jeffrey R. Wozniak, Ph.D., Department of Psychiatry, University of Minnesota, F256/2B West, 2450 Riverside Ave., Minneapolis, MN 55454; jwozniak@umn.edu; Phone: 612-273-9741; Fax: 612-273-9779.

Publisher's Disclaimer: This is a PDF file of an unedited manuscript that has been accepted for publication. As a service to our customers we are providing this early version of the manuscript. The manuscript will undergo copyediting, typesetting, and review of the resulting proof before it is published in its final citable form. Please note that during the production process errors may be discovered which could affect the content, and all legal disclaimers that apply to the journal pertain.

Conflict of Interest Statement

The authors have no competing interests. 


\section{Introduction}

Fetal alcohol spectrum disorders (FASD) is the largest known cause of intellectual disability [1], and children with FASD exhibit life-long cognitive deficits and behavior problems [24]. FASD is a serious public health problem, affecting as many as $2-5 \%$ of young children in the U.S. [17]. Effective interventions for these cognitive and behavioral deficits in FASD are needed.

There are no biological treatments for the neurodevelopmental deficits in FASD. However, animal models show that prenatal and postnatal dietary supplementation with specific nutrients attenuates neurodevelopmental damage and improves cognitive outcomes in offspring with prenatal alcohol exposure [11,22,25-27]. Translating pre-clinical nutritional findings for interventions in human children presents several challenges, one of which is controlling for the potentially confounding effects of dietary intake. Normal dietary intake needs to be considered in determining the most effective and tolerable dosages for individual nutrients and combinations of nutrients. Attention should be paid to nutrients that have particularly large effects on early brain development $[8,20]$ and are likely to be in deficit in the FASD population. Identifying the latter was the goal of the current study.

In animal studies, supplementation is typically added to a standard adequate diet. These studies assess the "added value" of supplemented nutrients. If diets of children with FASD are deficient in key nutrients, a potential treatment would need to not only correct the dietary deficiency but also provide additional supplementation for potential neurodevelopmental benefit. The dietary requirements of many nutrients follow a U-shaped risk curve, with risks for adverse outcomes highest with either deficient or excessive intakes [12]. The most effective and safest dose will need to provide supplementation without exceeding tolerable upper limits. Moreover, nutrient-nutrient interactions must be considered because they affect bioavailability (absorption and utilization). For instance, choline and folate are involved in methyl metabolism, and choline requirements increase during a folate deficiency [13]. Another example is that high doses of iron supplementation decrease zinc bioavailability [12]. The fact that this is generally only seen with iron supplementation and not with normal iron levels from a typical diet emphasizes the need for particular attention when supplementing with large doses of a nutrient.

Currently, the adequacy of dietary intake is not known in FASD. Anecdotally, children with FASD are often "picky eaters", some have autistic-like taste and texture sensitivities, and many have behavioral challenges such as rigidity and oppositionality. Also, many children with FASD come from impoverished environments affected by alcoholism - environments in which nutrition was inadequate and mealtimes were inconsistent and unpredictable (factors that contribute to behavioral dysregulation around eating). These factors can result in inadequate and unbalanced intakes of nutrients key for brain development and function.

The goal of this study was to evaluate the dietary intake of a representative sample of children with FASD in the age range where supplementation might be beneficial in order to determine the adequacy of intake of nutrients critical to brain development.

\section{Methods}

\subsection{Participants}

Participants included 31 children with FASD, ages 2.5 to 4.9 years at enrollment, who were in a 9-month study of choline supplementation. Children were recruited from a University FASD Clinic and an International Adoption Clinic (Table 1). Twenty-six participants were living with adoptive families (6 international; 20 domestic), and five were with biological 
relatives. None were living with biological parents. The study took place between September, 2009, and January, 2013. As part of clinical evaluations, children were seen by a pediatric psychologist and a pediatrician with formal training in the University of Washington diagnostic system for FASD [3]. Data on growth, facial dysmorphology, cognition, and alcohol exposure were collected.

We applied modified Institute of Medicine (IOM) criteria [10] to data from clinic and the baseline visit. If more than one growth, facial, or cognitive measurement was available, the most severe measure was used. For FAS, criteria require dysmorphic facial features, growth deficiency, and deficient brain growth. For Partial FAS, a dysmorphic face and either of the following were required: growth deficiency or central nervous system (CNS) impairment as defined by deficient brain growth or cognitive/behavioral impairment. For alcohol-related neurodevelopmental disorder (ARND), confirmed exposure was required with CNS impairment (deficient brain growth and/or cognitive/behavioral impairment). Of 31 participants, 7 (22.6\%) met criteria for FAS; 12 (38.7\%) for Partial FAS; and 12 (38.7\%) for ARND.

Growth deficiency was defined as height or weight $\leq 10 \%$ ile [15]; deficient brain growth was defined as structural abnormalities and/or OFC $\leq 10 \%$ ile [29]; a dysmorphic face had at least two features: short palpebral fissures ( $\leq 10 \%$ ile) [9], thin upper lip (rank 4 or 5 [3]), and smooth philtrum (rank 4 or 5 [3]). Because IOM criteria do not quantify cognitive/ behavioral deficits, we applied Centers for Disease Control and Prevention (CDC) criteria, specifying either global cognitive impairment $>2$ standard deviations (SD) or deficits of $>1$ $\mathrm{SD}$ in three or more domains (e.g., intellectual, language, motor, visual-perceptual, adaptive, behavioral) [4]. Of 31 participants, 7 (35\%) met CNS criteria based on brain growth, 20 (10\%) had global cognitive impairment, and 19 (95\%) had deficits in three or more domains (1 did not have sufficient cognitive testing to judge this criteria, but met CNS criteria for deficient brain growth). Seven participants (35\%) met two or more CNS criteria.

Of 31 participants, 23 (74\%) had confirmed prenatal alcohol exposure, including self-report by the biological mother or social service records indicating heavy maternal use during pregnancy. Inclusion required alcohol use consistent with Rank 3 or 4 in the Washington system [3]. Examples included daily use throughout pregnancy, use to intoxication every other day, or maternal intoxication at delivery. Minimal use, (e.g. occasional consumption of $<4$ drinks, or without intoxication) did not meet criterion. Eight participants had only "suspected" alcohol exposure, but met the criteria for FAS $(n=1)$ or partial FAS $(n=7)$. All 8 had dysmorphic faces and cognitive deficits as defined. In 22 cases, prenatal drug use was suspected. In all cases, alcohol was the predominant substance of abuse and use was extensive.

Exclusion criteria were presence of a developmental disorder (ex. Autism, Down Syndrome), neurological disorder, traumatic brain injury, or other medical condition affecting the brain. Psychiatric co-morbidity, such as attention deficit hyperactivity disorder (ADHD), was not exclusionary as co-morbidity is high in FASD [21]. All but one participant (a twin born at 36 weeks weighing 1360 grams) had a birthweight $>1500$ grams.

All procedures were approved by a University IRB and all participants underwent an informed consent procedure.

\subsection{Dietary Intake}

Dietary intake data were collected three times during the 9-month study: at baseline, 6months, and 9-months into the study. Data were collected via interview-administered 24hour recalls using the Automated Self-Administered 24-hour Recall (ASA24) [19]. A 
research assistant interviewed participants' caregivers and entered dietary data into ASA24 during study visits. These data were collected during a trial of choline supplementation in FASD. Only nutrient intake from food was included in the analysis; supplements, including the choline supplement, were not included.

Primary measures included dietary intake of macronutrients and 17 vitamins/minerals. Mean values for each nutrient were created from the three time points to create an observed daily nutrient intake. Most participants $(n=20)$ had three data points. For those with fewer data points, means were from two dietary recalls $(n=6)$ or a single dietary recall $(n=5)$.

Observed nutrient intakes were compared using one-sample $t$-tests to national dietary intake data from 24-hour dietary recalls of children ages 2-5 years (What we Eat in America, National Health and Nutritional Examination Survey [NHANES] 2007-2008 [28]). The dietary recalls for this study and for NHANES 2007-2008 used similar collection and processing methods. Both were collected using the U.S. Department of Agriculture's (USDA) Automated Multiple-Pass Method during interview-administered 24-hour recalls. Both datasets used the USDA's Food and Nutrition Database for Dietary Studies, 4.1 (FNDDS 4.1) [2] to process the dietary intakes. Therefore the two datasets could be compared to identify differences in dietary intake between children with FASD and similarage children. Although NHANES data were derived from single day dietary reports, daily variation in intake was addressed by the inclusion of a large sample $(\mathrm{N}=832)$ and by calculating nutrient totals using sample weights to account for differences in the day of the week of the dietary recall. In the current study, we utilized three independent datapoints, obtained over the course of the 9-month study, in order to address daily variability and to increase the reliability of our measures.

To evaluate whether the observed nutrient intakes were adequate, data were compared to the Dietary Reference Intakes (DRIs) [12]. DRIs are dietary recommendations established for a given age range to meet or exceed the requirements of the majority of healthy children within that age range. However, because there is individual variability in nutrient requirements, the IOM provides guidelines [12]. The Recommended Dietary Allowance (RDA) meets the nutritional needs of 97-98\% of individuals and is the recommended intake. Therefore, observed nutrient intakes above the RDA are considered likely adequate. The Estimated Average Requirement (EAR) represents the median nutrient requirement, or the intake that is adequate for 50\% of individuals in a group. Observed nutrient intakes below the EAR are considered likely inadequate. Conclusions about the adequacy of observed intakes between the EAR (the adequate intake for $50 \%$ of individuals) and RDA (the adequate intake for 97-98\%) cannot be made; however, observed intakes in this range are considered likely sub-optimal [12]. The EAR and RDA have not been established for several nutrients due to insufficient data. For these nutrients, the adequate intake (AI) represents a level that likely exceeds the actual (however unknown) requirements. Observed intakes above the AI are considered likely adequate. We report only those nutrients for which the majority of our sample ( $>50 \%)$ did not meet the RDA or AI.

\subsection{Physical Growth}

A physical examination was completed at baseline. Height-for-age $z$-score (HAZ), weightfor-age $z$-score (WAZ), and weight-for-height $z$-score (WHZ) were calculated using EpiInfo version 3.5.4 [5] with reference to CDC 2000 data for growth comparisons to U.S. children. 


\section{Results}

Although children with FASD had comparable dietary intakes to similar-age children on many nutrients, there were significant differences (Table 2). Children with FASD had lower intakes of saturated fats, vitamin $\mathrm{D}$, and calcium.

Based on the DRIs (Table 2), there was inadequate intake among children with FASD for macronutrients, vitamins, and minerals. The majority of children did not have likely adequate intakes ( $>\mathrm{AI})$ of total dietary fiber or poly-unsaturated fatty acids (PUFAs). Specifically, most children (90\%; $n=28)$ did not meet the AI for total dietary fiber. Only one participant (3\%) had adequate intake (>AI) of $\mathrm{n}-3$ fatty acids, and only half $(52 \% ; n=16)$ had adequate intakes (>AI) of n- 6 fatty acids.

In addition, inadequate intake was likely for 5 of 17 vitamins and minerals examined. The majority had likely inadequate intakes ( $\angle E A R)$ of vitamins D and E. Specifically, none of the participants met the EAR $(10 \mu \mathrm{g} / \mathrm{d})$ for vitamin D. For vitamin E, $74 \%(n=23) \mathrm{did}$ not meet the EAR (1-3yrs: $5 \mathrm{mg} / \mathrm{d}$; $4-8 \mathrm{yrs}: 6 \mathrm{mg} / \mathrm{d}$ ), and only $13 \%(n=4)$ had likely adequate intakes (>RDA). The majority did not have likely adequate intakes (>AI) of either vitamin $\mathrm{K}$ or choline; $65 \%(n=20)$ did not meet the AI for vitamin $\mathrm{K}$ and $84 \%(n=26)$ did not meet the AI for choline. Calcium was the only mineral of which the majority did not have likely adequate intakes (>RDA). Seventy-four percent $(n=23)$ did not meet the RDA for calcium, with $35 \%(n=11)$ having likely inadequate intakes below the EAR (1-3yrs: $500 \mathrm{mg} / \mathrm{d} ; 4$ $8 \mathrm{yrs}: 800 \mathrm{mg} / \mathrm{d})$. Although the majority had likely adequate intakes of iron, $32 \%(n=10)$ did not meet the RDA.

Although dietary supplements were not included in the analysis, 52\% ( $n=16)$ reported giving their child at least one daily supplement at any time during the study. Daily supplements included multivitamins $(48 \% ; n=15)$, omega-3 or fish oil $(13 \% ; n=4)$, vitamin $\mathrm{D}(7 \% ; n=2)$, calcium $(7 \% ; n=2)$, and iron $(3 \% ; n=1)$. Parents were asked to not add supplements containing choline and no parent reported doing so during the study. After each dietary recall, parents were asked whether food amounts reported were typical. Most (87\%) were described as typical, $10 \%$ were atypically low, and $3 \%$ were atypically high.

One sample $t$-tests compared the sample mean HAZ, WAZ, and WHZ to the U.S. reference population $(M=0)$. Our sample means did not differ from the reference population (Table 1) for HAZ, $t(30)=-1.37, p=0.182$, WAZ, $t(30)=-.35, p=0.732$, or $\mathrm{WHZ}, t(30)=0.72, p=0.478$.

To determine whether our dietary intake results were disproportionately affected by the growth deficient children in our sample having lower nutrient intakes, the four participants who were growth deficient were removed from analyses, and the results did not change. There was no association between either age or gender and observed nutrient intakes. Finally, given that the dietary intake data were collected as part of a choline intervention study, we examined whether participants changed their diets over the duration of the study. Repeated measures ANOVA revealed no change in dietary choline over the three dietary recalls, $\mathrm{F}(2,38)=0.564, p=.574$. Although the study did not have the statistical power to assess for potential changes in all of the nutrients of interest over the course of the study, it is re-assuring that there were no changes in the principal nutrient under investigation.

\section{Discussion}

To our knowledge, this is the first study reporting on dietary intake of children with FASD. Although they had similar caloric intakes to same-age children (NHANES), they had lower consumption of saturated fats, vitamin D and calcium compared to NHANES, a pattern that suggests they may be consuming fewer dairy products than similar-age children. Although 
deficient growth is a feature of FASD, our sample did not differ significantly in height or weight from U.S. children; therefore, their dietary requirements should not differ from U.S. standards.

Children with FASD did not meet the recommended intakes for several nutrients, and have a dietary pattern that could benefit from improving intakes of dairy products, green leafy vegetables, vegetable oils and nuts, as well as eggs and fish. Most (>50\%) did not meet the AI for fiber, $\mathrm{n}-3$ fatty acids, vitamin $\mathrm{K}$, or choline, or the RDA for vitamin $\mathrm{D}$, vitamin $\mathrm{E}$ or calcium. Specifically, only one child met the AI for n-3 fatty acids, all had inadequate vitamin $\mathrm{D}$, and $84 \%$ did not meet the AI for choline. Almost half did not meet the AI for $\mathrm{n}-6$ fatty acids and nearly one-third were not meeting the RDA for iron. Other than calcium and vitamin $\mathrm{D}$, the intakes of these nutrients were similar to NHANES data, suggesting that low intakes of these nutrients are not unique to children with FASD and may reflect a typical American diet among children that includes many low-nutrient, energy-dense foods and limited fruits, vegetables, and whole grains [6,7].

This diet profile in FASD, in which the intake does not meet adequacy for several nutrients critical to brain development, suggests that nutritional intervention may have potential as a component of treatment for these children. Several of the nutrients for which there was not adequate intake have demonstrated relevance to cognition and behavior in pre-clinical studies of FASD. For instance, supplementation in prenatally exposed animals with postnatal choline attenuates memory deficits and reduces excess activity [22,25-27]; vitamin D improves behavioral flexibility [11]; and $n-3$ fatty acids reduce oxidative damage in hippocampus, prefrontal cortex (PFC), and cerebellum [22]. Other nutrients may also have potential. For instance, nearly one-third of our sample did not meet the RDA for iron, a nutrient that is important in early brain development - including postnatal brain development [16].

The inadequate dietary patterns that we measured in children with FASD suggest that human nutritional supplementation trials in FASD should incorporate doses that will both overcome inadequate intakes and provide additional supplementation beyond the DRI. Preclinical data suggest that there may be neurocognitive benefits of supplementation beyond merely achieving sufficiency (typically, pre-clinical studies compare nutrient supplementation to a control group fed a standard/adequate diet [11,22]). These benefits may have their effect through two potential pathways in humans. The nutrient supplement may (1) change the course or pathophysiology of FASD at the basic biochemical level (e.g., epigenetic modification through choline supplementation) or (2) provide scaffolding for neurodevelopment by ensuring that there is no additional pathology from nutrient deficits.

Adequate nutrition is critical during prenatal and early postnatal development when the CNS is undergoing rapid changes $[8,20]$. In this study, we examined nutritional intake in children ages 2.5 to 5 years. Prior to age 2 years, neurodevelopment is particularly active with peak synaptogenesis and significant brain growth occurring by this age [20], and adequate nutrition and supplementation may have its largest benefits during this time. However, cortical synaptogenesis and myelination continue beyond age 2, particularly in regions of higher cognitive functioning (e.g, PFC), and these neurodevelopmental processes may be supported by nutrients such as choline and n-3 fatty acids which were low in our sample [20]. Clearly, understanding patterns of nutrient intake among children with FASD at different ages will be of added benefit. It will be important to determine the optimal "windows" during which nutritional interventions will be most effective. As one example, the window for choline in rat brain development appears to include gestation and at least the first 30 postnatal days [18], corresponding to the first several years of human childhood. In contrast, prenatal deficiencies of iron or zinc exacerbate the effects of prenatal alcohol 
exposure, suggesting that adequate amounts of these nutrients during gestation play a protective role against the teratogenic effects at the time of exposure [14,23].

A potential limitation of the current study is the reliance on interview-administered 24-hour recalls as opposed to extensive food diaries. However, these data match the NHANES data, which were also 24-hour recall data. For most participants, we averaged data from three time points, increasing reliability. Another potential limitation relates to individual variability in nutrient requirements - making determinations of "adequacy" difficult; however the observed nutrient intakes were evaluated in accordance with the IOM recommendations for using DRIs [12].

In conclusion, the pattern of dietary intake we measured in children with FASD suggests that there are opportunities for nutritional intervention and highlights the need for clinical supplementation trials in neurodevelopmental disorders like FASD. Based on the developmental window during which various nutrients are effective in pre-clinical models, the fact that early human childhood represent a period of intense brain development, and evidence of inadequate intake of key nutrients, nutritional supplementation in young children appears to have significant potential for neurodevelopmental interventions in FASD.

\section{Acknowledgments}

This work was supported by the National Institutes of Health (5R21AA019580; R33AA019580; P30DK56350). The study sponsors had no role in the study design, the collection, analysis and interpretation of the data, the writing of the manuscript, or the decision to submit this manuscript for publication.

\section{References}

1. Abel EL, Sokol RJ. Incidence of fetal alcohol syndrome and economic impact of FAS- related anomalies. Drug Alcohol Depend. 1987; 19:51-70. [PubMed: 3545731]

2. Agricultural Research Service - Food Surveys Research Group. USDA Food and Nutrient Database for Dietary Studies, 4.1. Beltsville, MD: 2010.

3. Astley, SJ. Diagnostic guide for fetal alcohol spectrum disorders: the 4-digit diagnostic code. 3rd Edition. Seattle, WA: University of Washington; 2004.

4. Centers for Disease Control Prevention. Guidelines for identifying and referring persons with Fetal Alcohol Syndrome. Morbidity and Mortality Weekly Report. 2005; 54:1-15. [PubMed: 15647722]

5. Dean, AG.; Sullivan, K.; Arner, TG.; Sangam, S.; Sunki, G.; Friedman, R.; Lantinga, M.; Zubieta, J.; Smith, DC. Epi Info 2000, a database and statistics program for public health professionals for use on Windows 95, 98, and NT computers. Atlanta, GA: Centers for Disease Control; 2000.

6. Ford CN, Slining MM, Popkin BM. Trends in Dietary Intake among US 2- to 6-Year- Old Children 1989-2008. Journal of the Academy of Nutrition and Dietetics. 2013; 113:35-42. e6. [PubMed: 23260722]

7. Fox MK, Condon E, Briefel RR, Reidy KC, Deming DM. Food Consumption Patterns of Young Preschoolers: Are They Starting Off on the Right Path? Journal of the American Dietetic Association. 2010; 110:S52-S59. [PubMed: 21092769]

8. Fuglestad, AJ.; Rao, R.; Georgieff, MK. The role of nutrition in cognitive development. In: Nelson, CA.; Luciana, M., editors. Handbook of developmental cognitive neuroscience. 2nd Edition. Cambridge: MIT Press; 2008. p. 623-641.

9. Hall, J.; Froster-Iskenius, U.; Allanson, J. Handbook of Normal Physical Measurements. Oxford University Press; 1989.

10. Hoyme HE, May PA, Kalberg WO, Kodituwakku P, Gossage JP, Trujillo PM, Buckley DG, Miller JH, Aragon AS. N. Khaole and others A practical clinical approach to diagnosis of fetal alcohol spectrum disorders: clarification of the 1996 institute of medicine criteria. Pediatrics. 2005; 115:39-47. [PubMed: 15629980] 
11. Idrus NM, Happer JP, Thomas JD. Cholecalciferol attenuates perseverative behavior associated with developmental alcohol exposure in rats in a dose-dependent manner. The Journal of Steroid Biochemistry and Molecular Biology.

12. Institute of Medicine, Dietary reference intakes: The essential guide to nutrient requirements. Washington, D.C: The National Academies Press; 2006.

13. Jacob RA, Jenden DJ, Allman-Farinelli MA, Swendseid ME. Folate Nutriture Alters Choline Status of Women and Men Fed Low Choline Diets. The Journal of Nutrition. 1999; 129:712-717. [PubMed: 10082779]

14. Keen CL, Uriu-Adams JY, Skalny A, Grabeklis A, Grabeklis S, Green K, Yevtushok L, Wertelecki WW, Chambers CD. The plausibility of maternal nutritional status being a contributing factor to the risk for fetal alcohol spectrum disorders: The potential influence of zinc status as an example. Biofactors. 2010; 36:125-135. [PubMed: 20333752]

15. Kuczmarski, RJ.; Ogden, CL.; Grummer-Strawn, LM.; Flegal, KM.; Guo, SS.; Wei, R.; Mei, Z.; Curtin, LR.; Roche, AF.; Johnson, CL. Adv Data. CDC growth charts: United States; 2000. p. $1-27$.

16. Lozoff B, Beard J, Connor J, Barbara F, Georgieff M, Schallert T. Long-lasting neural and behavioral effects of iron deficiency in infancy. Nutr Rev. 2006; 64:S34-S43. discussion S72-91. [PubMed: 16770951]

17. May PA, Gossage JP, Kalberg WO, Robinson LK, Buckley D, Manning M, Hoyme HE. Prevalence and epidemiologic characteristics of FASD form various research methods with an emphasis on recent in-school studies. Dev Disabil Res Rev. 2009; 15:176-192. [PubMed: 19731384]

18. Meck WH, Smith RA, Williams CL. Organizational changes in cholinergic activity and enhanced visuospatial memory as a function of choline administered prenatally or postnatally or both. Behav Neurosci. 1989; 103:1234-1241. [PubMed: 2610916]

19. National Cancer Institute, Automated Self-Administered 24-Hour Recall (ASA24)-Beta. Bethesda, MD: National Cancer Institute; 2009.

20. Nyaradi A, Li J, Hickling S, Foster J, Oddy WH. The role of nutrition in children's neurocognitive development from pregnancy through childhood. Frontiers in Human Neuroscience. 2013; 7

21. O'Connor MJ, Shah B, Whaley S, Cronin P, Gunderson B, Graham J. Psychiatric illness in a clinical sample of children with prenatal alcohol exposure. American Journal of Drug and Alcohol Abuse. 2002; 28:743-754. [PubMed: 12492268]

22. Patten AR, Brocardo PS, Christie BR. Omega-3 supplementation can restore glutathione levels and prevent oxidative damage caused by prenatal ethanol exposure. The Journal of Nutritional Biochemistry. 2012

23. Rufer ES, Tran TD, Attridge MM, FGR AME, S.S.M. Adequacy of maternal iron status protects against behavioral neuroanatomical and growth deficits in fetal alcohol spectrum disorders. PLoS One. 2012; 7:e47499-e47499. [PubMed: 23094056]

24. Sokol RJ, Delaney-Black V, Nordstrom B. Fetal alcohol spectrum disorder. Jama. 2003; 290:2996-2999. [PubMed: 14665662]

25. Thomas JD, Biane JS, O'Bryan KA, O'Neill TM, Dominguez HD. Choline supplementation following third-trimester-equivalent alcohol exposure attenuates behavioral alterations in rats. Behav Neurosci. 2007; 121:120-130. [PubMed: 17324056]

26. Thomas JD, Garrison M, O'Neill TM. Perinatal choline supplementation attenuates behavioral alterations associated with neonatal alcohol exposure in rats. Neurotoxicol Teratol. 2004; 26:3545. [PubMed: 15001212]

27. Thomas JD, La Fiette MH, Quinn VR, Riley EP. Neonatal choline supplementation ameliorates the effects of prenatal alcohol exposure on a discrimination learning task in rats. Neurotoxicol Teratol. 2000; 22:703-711. [PubMed: 11106863]

28. U.S. Department of Agriculture - Agricultural Research Service. Nutrient Intakes from Food: Mean Amounts Consumed per Individual, by Gender and Age. What We Eat in America. 2010. NHANES 2007-2008. Available: www.ars.usda.gov/ba/bhnrc/fsrg) 
29. WHO Multicentre Growth Reference Study Group. WHO Child Growth Standards: Length/heightfor-age, weight-for-age, weight-for-length, weight-for-height and body mass index-for-age: Methods and development., 2006). 


\section{Highlights}

- Children with FASD have lower intakes of vitamin D and calcium compared to national dietary intake data from similar-age children.

- Children with FASD are not consuming recommended intakes of vitamin D, choline, and n-3 fatty acids, nutrients important for neurodevelopment.

- The dietary pattern in children with FASD highlights the potential for nutritional supplementation to provide scaffolding for improving neurobehavioral functioning. 
Table 1

Participant characteristics at baseline

\begin{tabular}{|c|c|}
\hline $\mathrm{N}(\%)$ or mean (SD) & $\mathbf{N}=\mathbf{3 1}$ \\
\hline Age (years) & $4.11(0.71)$ \\
\hline \multicolumn{2}{|l|}{ Gender } \\
\hline Male & $11(35.5 \%)$ \\
\hline Female & $20(64.5 \%)$ \\
\hline \multicolumn{2}{|l|}{ Anthropometry/physical growth } \\
\hline Height-for-Age $z$-Score & $-0.22(0.89)$ \\
\hline Weight-for-Age $z$-Score & $-0.07(1.10)$ \\
\hline Weight-for-Height $z$-Score & $0.13(1.01)$ \\
\hline \multicolumn{2}{|l|}{ Dysmorphic Facial Features } \\
\hline Lip (rank 4 or 5) & $13(42 \%)$ \\
\hline Philtrum (rank 4 or 5) & $15(48 \%)$ \\
\hline Palpebral Fissure ( $\Delta 0^{\text {th }}$ percentile $)^{a}$ & $19(61 \%)$ \\
\hline$\geq 2$ Facial Features Present & $13(42 \%)$ \\
\hline \multicolumn{2}{|l|}{ Growth Deficiency ( $\triangle O^{\text {th }}$ percentile) } \\
\hline Height & $3(10 \%)$ \\
\hline Weight & $4(13 \%)$ \\
\hline \multicolumn{2}{|l|}{ Deficient Brain Growth ( $\triangle 1 O^{\text {th }}$ percentile) } \\
\hline Occipital-Frontal Circumference & $3(10 \%)$ \\
\hline \multicolumn{2}{|l|}{ Alcohol/Drug Exposure } \\
\hline Alcohol Confirmed & $23(74 \%)$ \\
\hline Alcohol Suspected & $8(26 \%)$ \\
\hline Other Drug Exposure Suspected & $22(71 \%)$ \\
\hline \multicolumn{2}{|l|}{ IOM Diagnostic Category } \\
\hline FAS & $7(23 \%)$ \\
\hline Partial FAS & $12(39 \%)$ \\
\hline ARND & $12(39 \%)$ \\
\hline \multicolumn{2}{|c|}{ Baseline Cognitive Functioning (Mullen Scales ${ }^{b}$ ) } \\
\hline Visual Reception & $46.8(15.3)$ \\
\hline Fine Motor & $42.4(13.2)$ \\
\hline Receptive Language & $43.5(10.9)$ \\
\hline Expressive Language & $41.4(9.1)$ \\
\hline Early Learning Composite & $88.1(18.9)$ \\
\hline
\end{tabular}

a Missing for one participant from whom an accurate measure could not be obtained ( $n=30)$.

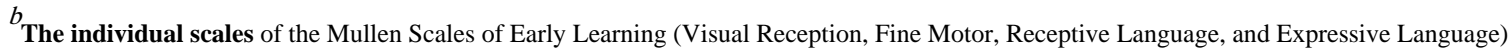
use a $t$-score distribution $(M=50 S D=10)$. The Early Learning Composite Score is on a scale with a mean of $100(S D=15)$. 


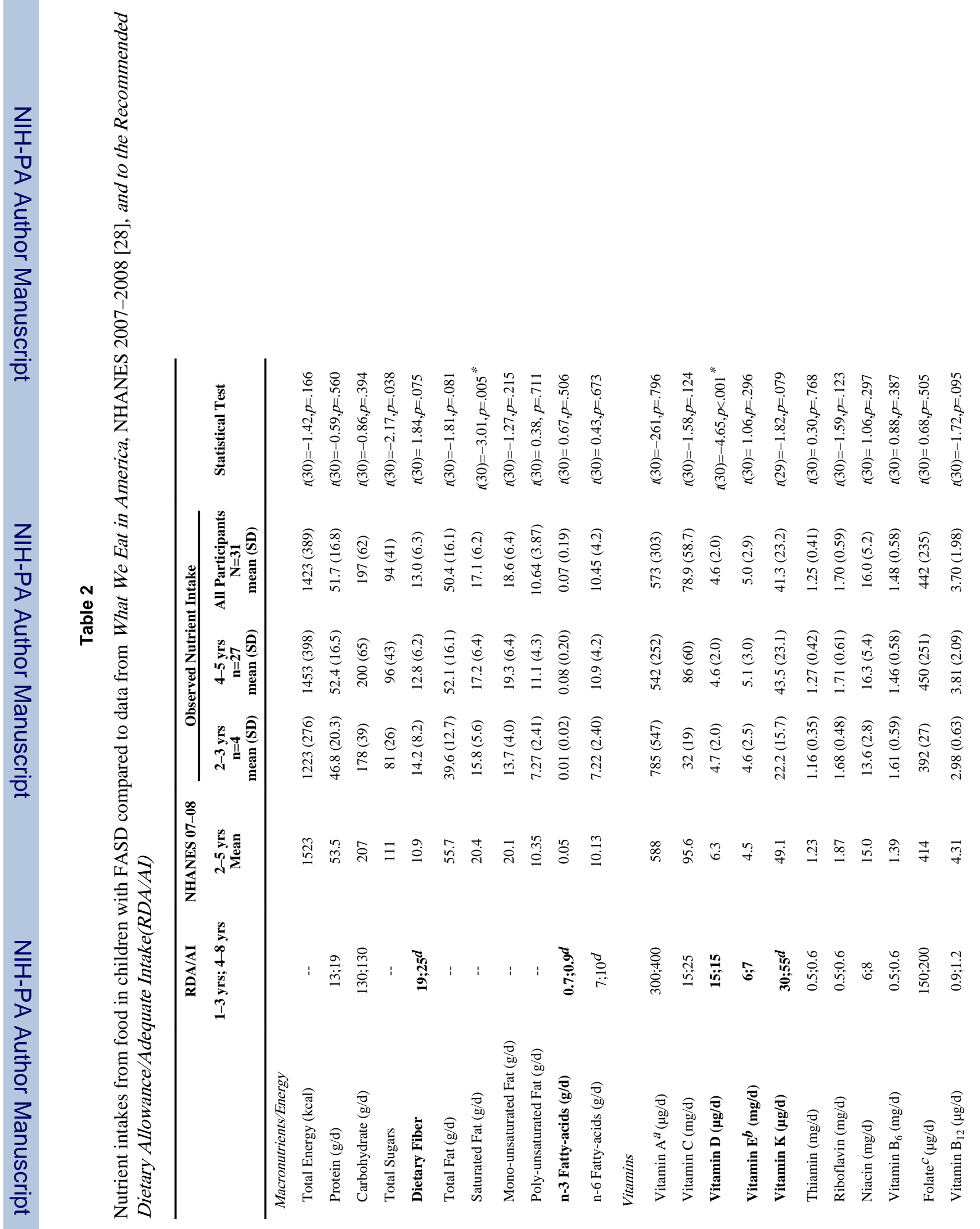




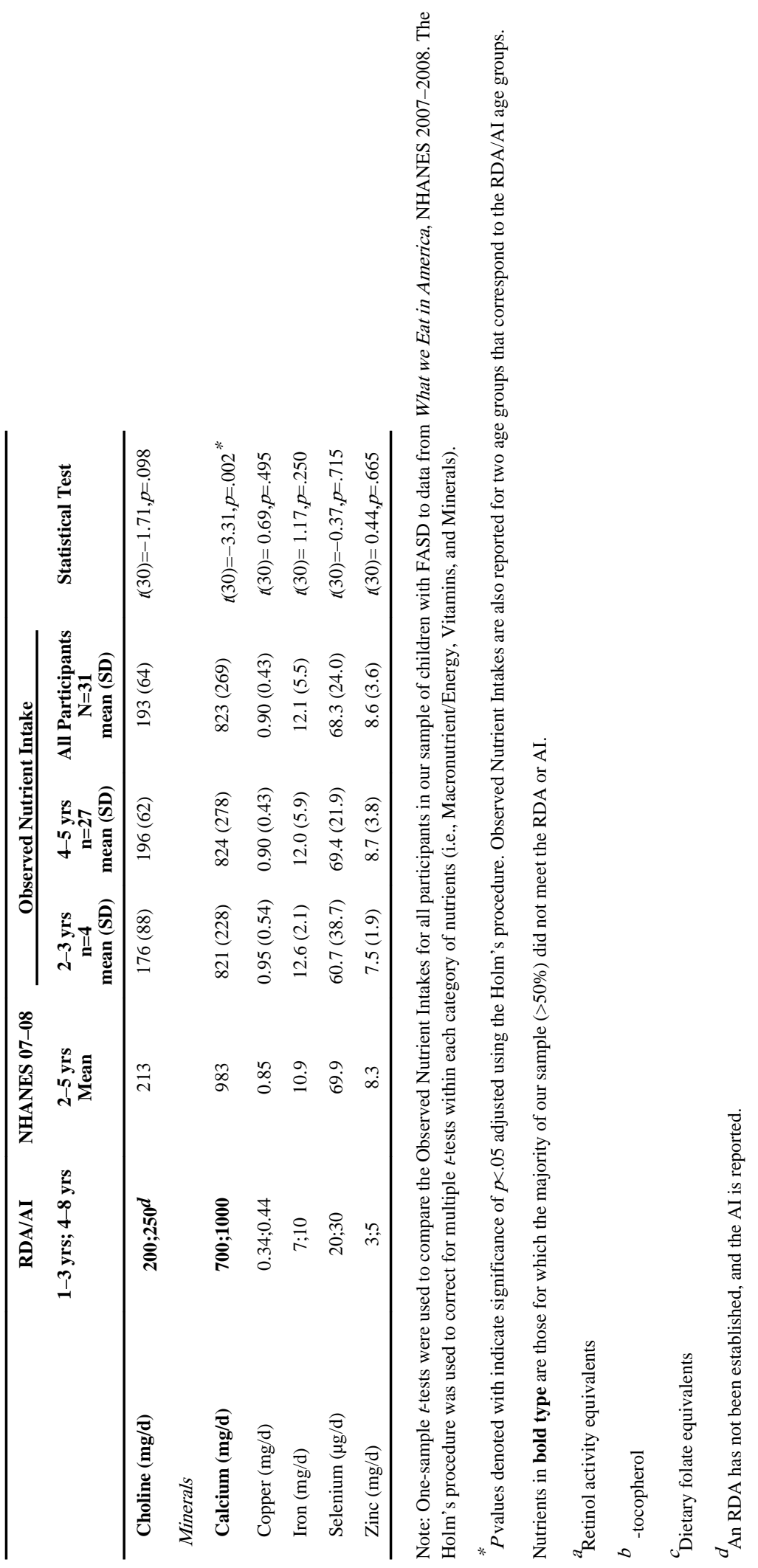

